\title{
NORMAL TRANSFORMATION SEMIGROUPS
}

\author{
J. S. V. SYMONS \\ (Received 20 February 1973) \\ Communicated by G. B. Preston
}

\section{Introduction}

Many transformation semigroups $S$ over a set $X$ have the inner-automorphism property: their automorphisms are precisely those mappings of the form $g^{-1} \cdot g: \alpha \rightarrow g^{-1} \alpha g(\alpha \in S)$ where $g$ is a fixed permutation of $S$. For example, the full transformation semigroup, any two sided ideal of this semigroup, and, with some exceptions for small cardinals, the alternating group and the symmetric group itself. See Malcev (1952) and Scott (1965, Chapter 11). In this paper we determine all transformation semigroups over finite $X$ with this property. In fact, we do more: we characterize all transformation semigroups invariant under the mappings $g^{-1} \cdot g$. We refer to these as $\mathscr{G}$-normal transformation semigroups.

\section{Notation, definitions}

If $X$ is a set we write $\mathscr{T}(X)$ for the semigroup of mappings from $X$ to $X$, under the operation of composition. As in Clifford and Preston (1967, page 241), we shall use the notation

$$
\alpha=\left(\begin{array}{c}
X_{i} \\
x_{i}
\end{array}\right)
$$

to mean that $\alpha$ in $\mathscr{T}(X)$ has range $\left\{x_{i} ; i \in I\right\}$ and $X_{i}=x_{i} x^{-1}$, for each $i$ in $I$. We refer to the set $\left\{X_{i} ; i \in I\right\}$ as the partition of $X$ induced by $\alpha$; the $X_{i}, i \in I$, are called the partition classes of $\alpha$.

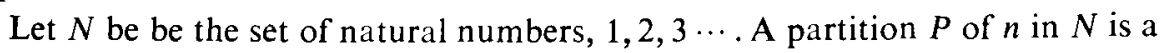
system of natural numbers $\left(n_{1}, n_{2}, \cdots, n_{k}\right)$ with $\sum_{i} n_{i}=n$, order being immaterial. We call $n_{1}, n_{2}, \cdots, n_{k}$ the terms of $P$, and define $\mathscr{P}(P)$ as the set of all partitions of $n$ obtained by adding together various terms of $P$. For example if $n=5$ and $P=(2,2,1)$ then $\mathscr{P}(P)=\{(2,2,1),(3,2),(4,1),(5)\}$.

If $\alpha$ belongs to $\mathscr{T}(X)$ where $|X|=n$ and $P$ is a partition of $n$, then $\alpha$ is of type $P$ if the terms of $P$ correspond bijectively to the cardinalities of the partition classes of $\alpha$. For example if $X=\{1,2,3,4,5\}$ and 


$$
\alpha=\left(\begin{array}{ccc}
\{1,2,3\} & \{4\} & \{5\} \\
1 & 2 & 3
\end{array}\right),
$$

then $\alpha$ is of type $(3,1,1)$. We put

$$
\mathscr{T}(X ; P)=\bigcup_{Q \equiv \mathscr{T}(P)}\{\alpha \in \mathscr{T}(X) ; \quad \alpha \text { is of type } Q\} .
$$

If $\alpha \in \mathscr{T}(X ; P)$ we shall say $\alpha$ is of class $P$. We note that a transformation has only one type, but may be of many classes: $\alpha$ above is of class $(2,1,1,1)$ and $(1,1,1,1,1)$ as well as $(3,1,1)$. However if $\alpha$ is of class $P$ and $|X \alpha|=|P|$, then $\alpha$ is of type $P$.

\section{Results}

In what follows the set $X$ is assumed to be finite with $|X|=n$. We derive our result from a series of lemmas.

LEMMA 1. For all partition, $P$, the semigroup $\mathscr{T}(X ; P)$ is a right ideal of $\mathscr{T}(X)$.

The $n$-termed partition $(1,1, \cdots, 1)$ will be abbreviated to $(1)$. Clearly $\mathscr{T}(X ;(1))$ $=\mathscr{T}(X)$.

Lemma 2. If $P \neq(1)$, each idempotent of $\mathscr{T}(X)$ of class $P$ is a product of idempotents of type $P$.

Proof. The idempotents of $\mathscr{T}(X)$ are precisely those mappings of the form

$$
\varepsilon=\left(\begin{array}{lll}
X_{1} X_{2} & \ldots & X_{k} \\
x_{1} x_{2} & & x_{k}
\end{array}\right)
$$

where $x_{i} \in X_{i}$. If $\varepsilon$ is of class $P$ then $X_{1}, X_{2}, \cdots, X_{k}$ has a refinement $Y_{1}, Y_{2}, \cdots, Y_{j}$ where $P=\left(\left|Y_{1}\right|,\left|Y_{2}\right|, \cdots,\left|Y_{j}\right|\right)$. For each $i \leqq j$ let $y_{i}$ be an arbitrary element of $Y_{i}$ and consider

$$
\eta_{1}=\left(\begin{array}{llll}
Y_{1} Y_{2} & \ldots & Y_{j} \\
y_{1} y_{2} & & y_{j}
\end{array}\right) .
$$

Now $\eta_{1}$ is of type $P$ and since $P \neq(1), \eta_{1}$ is neither injective nor surjective. Taking $z$ not in $X \eta_{1}$ it follows that the system

$$
\left\{y_{1}, y_{2}\right\}, z, y_{3}, y_{4}, \cdots, y_{j}
$$

may be extended to a partition of $X$

$$
Z_{1}, Z_{2}, \cdots, Z_{j}
$$

where each $Z_{i}$ contains the corresponding set or element of the former sequence, 
and, moreover, $P=\left(\left|Z_{1}\right|,\left|Z_{2}\right|, \cdots,\left|Z_{j}\right|\right)$. (We exclude the possibility $P=(n)$. In this case $\varepsilon$ is a constant function and the result is trivial). We set

$$
\eta_{2}=\left(\begin{array}{lll}
Z_{1} Z_{2} & Z_{j} \\
y_{1} z & \cdots & y_{j}
\end{array}\right) \text {. }
$$

Now $\eta_{2}$ is of type $P$ and

$$
\eta_{1} \eta_{2}=\left(\begin{array}{ccccc}
Y_{1} \cup & Y_{2} & Y_{3} & & Y_{j} \\
y_{1} & y_{3} & & y_{j}
\end{array}\right)
$$

Since each $X_{i}, i \leqq k$, is a union of certain of the $Y_{i}$ the proof is completed by a judicious selection of the $y_{i}$, and an elementary induction argument. We omit the details.

Lemma 3. If $P \neq(1)$ then $\mathscr{T}(X ; P)$ is generated by its idempotents of type $P$.

Proof. We show each $a \in \mathscr{T}(X ; P)$ of type $Q$ may be written as a product of idempotents of type $Q$, and the result follows from Lemma 2 . ing properties:

Let $\alpha$ be of type $Q, \alpha=\left(\begin{array}{llll}X_{1} & X_{2} & \ldots & X_{k} \\ x_{1} & x_{2} & & x_{k}\end{array}\right)$. Choose $\beta$ in $\tilde{J}(X)$ with the follow-
properties:

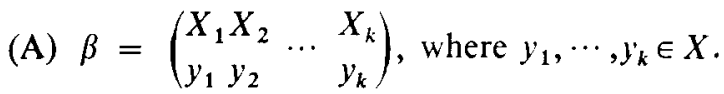

(B) $\beta$ is a product of idempotents of type $Q$.

(C) If $\gamma$ has properties (A) and (B) then

$$
m=\left|\left\{X_{i} ; \quad X_{i} \beta=X_{i} x\right\}\right| \geqq\left|\left\{X_{i} ; X_{i} \gamma=X_{i} x\right\}\right| .
$$

We show $m=k$. Assume by way of contradiction that $m<k$. For convenience we renumber the $X_{i}$ so that $x_{i}=y_{i}$ if and only if $i \leqq m$. (We admit the possibility $m=0$ and interpret this in the obvious sense.) There are two cases to consider.

Case $1: x_{m+1} \notin X \beta$. Then as in Lemma 2 the system

$$
y_{1}, \cdots, y_{m},\left\{y_{m+1}, x_{m+1}\right\}, y_{m+2}, \cdots, y_{k}
$$

may be extended to a partition $Y_{1}, Y_{2}, \cdots, Y_{k}$ of $X$ where $Q=\left(\left|Y_{1}\right|,\left|Y_{2}\right|, \cdots,\left|Y_{k}\right|\right)$. Put

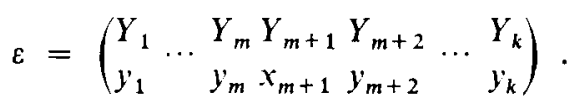

Clearly $\varepsilon$ is idempotent, and of type $Q$. Moreover the partition classes of $\beta \varepsilon$ are $X_{1}, \cdots, X_{k}$ and $X_{i} \beta \varepsilon=y_{i} \varepsilon=y_{i}$ for $i \leqq m$, while $X_{m+1} \beta \varepsilon=y_{m+1} \varepsilon=x_{m+1}$ $=X_{m+1} \alpha$, contrary to choice of $\beta$. 
Case 2: $x_{m+1} \in X \beta, x_{m+1}=y_{m+2}$, say, (since $x_{m+1}$ cannot be any $y_{i}$ for $i \leqq m+1)$. Then choose $z \in X \beta$ and extend $y_{1}, \cdots, y_{m+1},\left\{z, y_{m+2}\right\}, y_{m+3}, \cdots, y_{k}$ to a partition $Y_{1}, \cdots, Y_{k}$ of $X$ of type $Q$. If we form

$$
\varepsilon=\left(\begin{array}{llllll}
Y_{1} & \ldots & Y_{m+1} & Y_{m+2} & Y_{m+3} & \ldots \\
y_{1} & & y_{m+1} & z & y_{m+3} & y_{k}
\end{array}\right)
$$

then

$$
\beta \varepsilon=\left(\begin{array}{lllllll}
X_{1} & \ldots & X_{m} & X_{m+1} & X_{m+2} & X_{m+3} & X_{k} \\
y_{1} & & y_{m} & y_{m+1} & z & y_{m+3} & y_{k}
\end{array}\right)
$$

and $x_{m+1} \notin X \beta \varepsilon$. We are now in Case 1 , and may proceed to a contradiction, as before.

Lemma 4. Let $S \leqq \mathscr{T}(X)$ be G-normal and let $\propto$ in $S$ be of type $P$. Then $S$ contains an idempotent of type $P$.

Proof. Let $\alpha \in S$ be of type $P$ and let $A$ be the sub-semigroup of $S$ generated by

$$
\left\{g^{-1} \alpha g ; g \in \mathscr{G}(X)\right\} \text {. }
$$

Choose $\beta \in A$ such that $\beta$ is of type $P$, and, of all the mappings in $A$ of type $P$, $\beta$ fixes a maximal number of elements of $X, k$ say. We put

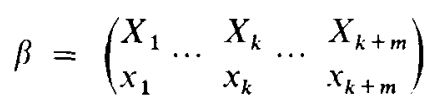

where $x_{i} \in X_{i}$ for $i \leqq k$, and it is sufficient to show $m=0$. Assume not, and let $R=\bigcup_{i=1}^{m} X_{k+i}$. If $R \subseteq X \beta$ then $R=X \beta$ where $Y \subseteq R$ (since $\beta$ maps $X \backslash R$ to $X \backslash R)$. It follows that $\beta \mid R$ is a permutation of $R$ so that $(\beta \mid R)^{p}=i_{R}$, for some $p$. Hence $\beta^{p}$ is idempotent, and of type $\left(\left|X_{1}\right|, \cdots,\left|X_{k}\right|, 1,1, \cdots, 1\right)$, which is evidently the type of $\beta$.

It follows that we may assume there exists some $z \in X_{k+1}$ (say) with $z \notin X \beta$. Let $y_{k+1}, \cdots, y_{k+m}$ be a sequence of representatives of $X_{k+1}, \cdots, X_{k+m}$. Observe that $x_{k+1} \notin X_{k+1}$. We define a partial transformation $g$ as follows

$$
\begin{aligned}
& x_{i} g \quad=x_{i} \quad(i \leqq k) \\
& x_{k+1} g=z \\
& z g=x_{k+1} \\
& x_{k+i} g=y_{k+i} \quad(2 \leqq i \leqq m)
\end{aligned}
$$

If $g$ is one to one we extend it to an element in $\mathscr{G}(X)$ and consider $g^{-1} \beta g \beta \in A$. Now $g^{-1} \beta g=\left(\begin{array}{l}X_{i} g \\ x_{i} g\end{array}\right)$ so $g^{-1} \beta g$ is of type $P$. Since $\mathscr{T}(X ; P)$ is a right ideal $g^{-1} \beta g \beta$ is of class $P$. On the other hand $X \beta g$ contains an element of each partition class of $\beta$, from which follows 


$$
\left|X g^{-1} \beta g \beta\right|=|X \beta g \beta|=|X \beta|,
$$

and we have proved that $g^{-1} \beta g \beta$ is of type $P$. However $x_{i} g^{-1} \beta g \beta=x_{i}(i \leqq k)$ and $x_{k+1} g^{-1} \beta g \beta=z \beta g \beta=x_{k+1} g \beta=z \beta=x_{k+1}$. Thus $g^{-1} \beta g \beta$ fixes at least $k+1$ elements of $X$, contrary to the choice of $\beta$.

If $g$ is not one to one we must have one of the $y_{k+i}$ coinciding with $x_{k+1}$. Let us say $x_{k+1}=y_{k+2}$. The $y_{k+i}$ were chosen merely as representatives of the $X_{k+i}$ and it may be possible to replace $y_{k+2}$ by some other element of $X_{k+2}$. If so, the former argument still goes through. Otherwise we are compelled to assume $X_{k+2}=\left\{x_{k+1}\right\}$. We define a map $h$ as follows:

$$
\begin{aligned}
& x_{i} h=x_{i} \quad(i \leqq k+1) \\
& x_{k+2} h=z \\
& x_{k+i} h=y_{k+i} \quad(i>2) .
\end{aligned}
$$

It will be readily checked that $h$ is one-to-one. As before we extend to $\omega(X)$ and consider $h^{-1} \beta h \beta$. This is of class $P$ and, by a similar argument to that above, of type $P$. Since it fixes the first $k+1$ of the $x_{i}$, we have reached our final contradiction. We conclude, $m=0$, and the result is proved.

If $\alpha=\left(\begin{array}{l}X_{i} \\ x_{i}\end{array}\right)$ and $\beta=\left(\begin{array}{l}Y_{i} \\ y_{i}\end{array}\right)$ are idempotents of the same type, it is easy to find $g \in \omega(X)$ such that the partition of $g^{-1} \propto g=\left(\begin{array}{l}X_{i} g \\ x_{i} g\end{array}\right)$ is $Y_{1}, Y_{2}, \cdots$. Hence if $g^{-1} \alpha g$ $=\left(\begin{array}{l}Y_{i} \\ z_{i}\end{array}\right)$ and we take $h \in \mathscr{G}(X)$ as the product of all the (disjoint) two-cycles $\left(z_{i}, y_{i}\right)$ then $(g h)^{-1} \alpha g h=h^{-1} g^{-1} \alpha g h=\left(\begin{array}{l}Y_{i} h \\ z_{i} h\end{array}\right)=\left(\begin{array}{l}Y_{i} \\ y_{i}\end{array}\right)=\beta$. It follows that if $S$ is $\mathscr{G}$-normal and contains one idempotent of a certain type it contains all idempotents of this type.

Corollary to Lemma 4 . With the assumptions of Lemma $4, S$ contains all idempotents of type $P$ and hence, by Lemma $3, S$ contains $\mathscr{T}(X ; P)$.

Lemma 5. Let $S \leqq \mathscr{T}(X)$ be $\mathscr{G}$-normal and assume $S \cap \mathscr{G}(X) \neq \square$. Then $S \cap \mathscr{G}(X)$ is a normal subgroup of $\mathscr{G}(X)$.

Proof. If $S \cap \mathscr{G}(X) \neq[\urcorner$, then it is a finite cancellative semigroup, and hence a group. Moreover, for all $g \in \mathscr{G}(X), g^{-1}(S \cap \mathscr{G}(X)) g=g^{-1} S g \cap g^{-1} \mathscr{G}(X) g$ $\subseteq S \cap \mathscr{G}(X)$, as required.

These last two results combine to show necessity below. Sufficiency is clear.

MAIN THEOREM. Let $S \leqq \mathscr{T}(X)$. Then $S$ is $\mathscr{G}$-normal if and only if $S$ is one of the following

(i) a normal subgroup of $\mathscr{G}(X)$

(ii) a union of semigroups of the form $\mathscr{T}(X ; P)$

(iii) a union of the two above. 
REMARK. If $S$ is $\mathscr{G}$-normal and is not a normal subgroup of $\mathscr{G}(X)$, then by this result $S$ contains all the constant functions on $X$. It is well known (see Malcev (1952)) that this ensures all automorphisms of $S$ are inner. Hence $S$ has the inner automorphism property. Thus we have: If $S$ is not contained in $\mathscr{G}(X)$, then $S$ is $\mathscr{G}$-normal if and only if it has the inner automorphism property.

\section{References}

A. H. Clifford and G. B. Preston (1967), The algebraic theory of semigroups, II (Math. Surveys of the American Math. Soc. 7, 1967).

A. I. Malcev (1952), 'Symmetric groupoids', Math. Sb., (N.S.) 31, 136-151.

W. R. Scott (1965), Group Theory (Prentice-Hall, New Jersey, 1965).

University of Western Australia,

Nedlands, W. A. 6009. 